Proyecciones Journal of Mathematics

Vol. 32, No 4, pp. 305-319, December 2013.

Universidad Católica del Norte

Antofagasta - Chile

\title{
A convergence result for unconditional series in
}

$$
L^{p}(\mu)^{*}
$$

Juan M. Medina and Bruno Cernuschi-Frías

Universidad de Buenos Aires, Argentina

Received: May 2012. Accepted : July 2013

\begin{abstract}
We give sufficient conditions for the convergence almost everywhere of the expansion with respect to an unconditional basis for functions in $L^{p} p \geq 2$. This result extends the classical theorem of Menchoff and Rademacher for orthogonal series in $L^{2}$.

Subjclass 2000 MSC Primary 42C15 ; Secondary: 46B15, 46B20.

Keywords : Unconditional basic sequence, almost sure convergence, random series.
\end{abstract}

\footnotetext{
${ }^{*}$ This work was partially supported by the Universidad de Buenos Aires, grant No. UBACyT 20020100100503, and the Consejo Nacional de Investigaciones Científicas y Técnicas, CONICET, Argentina
} 


\section{Introduction}

In this paper we study the almost everywhere convergence of unconditional series in a general $\sigma$-finite $L^{p}$ space. The main result of this work is a generalization to $L^{p}$ of the classical result of D. Menchoff and Rademacher ([1], [7],[9]) on the almost everywhere convergence of orthogonal series in $L^{2}$. Theorem 1.1 below is a generalization of that result. When $p=2$ and the series are orthogonal this contains the generalization obtained by Moricz and Tandori [10] (Theorem 1). The result of Menchoff has interesting implications in several areas of Analysis: Fourier Series ([1] [14]), and specially in Probability Theory ([2], [4], [7]) where this result is related to the laws of large numbers. On the other hand, unconditional basis are very important in Wavelet Analysis and related fields. One of the difficulties found when trying to generalize this result to $L^{p}, p \neq 2$, is the lack of the notion of orthogonality. Fortunately, it is not orthogonality what is needed but a consequence of it; uncondtional convergence. We will prove that this property together with another similar to the original used by Moricz and Tandori seem to be sufficient to ensure the almost everywhere convergence of certain expansions in $L^{p}$.

The main result can be stated formally:

Theorem 1.1. Let $\left\{f_{j}\right\}_{j \in \mathbf{N}}$ be a basic sequence in $L^{p}(X, F, \mu), p \geq 2$.

If for some $0<\epsilon \leq 2$ :

$\sum_{n=0}^{\infty} \sum_{j \in I_{n}} a_{j} \log (j)^{\frac{\varepsilon}{2}}\left(\log \frac{2 A_{n}^{2}}{a_{j}^{2}}\right)^{1-\frac{\varepsilon}{2}} f_{j}, \quad I_{n}=\left\{2^{n}+1, \ldots, 2^{n+1}\right\}, A_{n}^{2}=\sum_{i=1}^{n}\left|a_{i}\right|^{2}$.

converges in the norm of $L^{p}(X, F, \mu)$ then: $\sum_{k=1}^{\infty} a_{k} f_{k}(x)$ converges for almost all $x \in X[\mu]$.

\section{Some definitions and known results}

Recall that a Schauder basis or a basic sequence (a basis for a closed subspace) is call unconditional if it verifies one (and hence all) of the equivalences of the following proposition: 
Proposition 2.1. A basic sequence $\left\{x_{n}\right\}_{n \in \mathbf{N}}$ in a Banach space $B$ is unconditional if and only if one of the following conditions is fullfilled:

i) For every permutation $\pi$ of the integers the sequence $\left\{x_{\pi(n)}\right\}_{n \in \mathbf{N}}$ is a basic sequence (is a basis of $\operatorname{span}\left\{x_{n}\right\}_{n \in \mathbf{N}}$ ).

ii) For every subset of integers $\sigma$ the convergence of $\sum_{n=1}^{\infty} a_{n} x_{n}$ implies the convergence of $\sum_{n \in \sigma} a_{n} x_{n}$.

iii) The convergence of $\sum_{n=1}^{\infty} a_{n} x_{n}$ implies the convergence of $\sum_{n=1}^{\infty} b_{n} x_{n}$, whenever $\left|b_{n}\right| \leq\left|a_{n}\right|$.

iv) The convergence of $\sum_{n=1}^{\infty} a_{n} x_{n}$ implies the convergence of $\sum_{n=1}^{\infty} \theta_{n} a_{n} x_{n}$ where $\theta_{n}=\mp 1$ arbitrarely.

As a consequence of this proposition using the properties of the Rademacher functions, an alternative characterization can be given for unconditional basic sequences in the particular case of $\sigma$-finite $L^{p}$ spaces, [13]:

Theorem 2.1. Let $\left\{f_{j}\right\}_{j \in \mathbf{N}}$ be a basic sequence in $L^{p}(X, F, \mu)(1 \leq p \leq$ $\infty)$. Then it is unconditional if and only if there exist $A_{p}, B_{p}$ positive constants such that:

$$
\begin{aligned}
& A_{p}\left\|\left(\sum_{j}\left|a_{j} f_{j}\right|^{2}\right)^{\frac{1}{2}}\right\|_{L^{p}(X)} \leq\left\|\sum_{j} a_{j} f_{j}\right\|_{L^{p}(X)} \leq B_{p}\left\|\left(\sum_{j}\left|a_{j} f_{j}\right|^{2}\right)^{\frac{1}{2}}\right\|_{L^{p}(X)}, \\
& \forall \sum_{j} a_{j} f_{j} \in L^{p}(X, F, \mu) .
\end{aligned}
$$

This result gives a very useful characterization in terms of the equivalence of norms for $\operatorname{span}\left\{f_{j}\right\}_{j}$. This equivalence will be very important in the sequel, though we will use it several times without refering to it explicitly, as it will become clear from the context.

Our results rely on maximal inequalities, so we need to define several maximal operators:

Definition 1. Let $f \in L^{p}(X, F, \mu), f=\sum_{k=1}^{\infty} a_{k} f_{k}$, we define:

$$
\mathcal{M}^{d} f(x)=\sup _{N \in \mathbf{N}}\left|S_{2^{N}} f(x)\right|
$$


where, given $n \in \mathbf{N}, x \in X$ then $S_{n} f(x)=\sum_{k=1}^{n} a_{k} f_{k}$.

$$
\mathcal{M} f(x)=\sup _{k \in \mathbf{N}}\left|S_{k} f(x)\right|
$$

and

$$
\mathcal{M}_{N}^{\sharp} f(x)=\sup _{2^{N-1}<n<2^{N}}\left|\sum_{k=2^{N-1}+1}^{n} a_{k} f_{k}(x)\right|
$$

\section{Auxiliary Results}

In the following we will also suppose that $(X, F, \mu)$ is a $\sigma$-finite measure space. We will call absolute constants as $K, C, c, C_{p}$, etc. Logarithms are taken in base 2 . In our results $\left\{f_{j}\right\}_{j}$ constitutes an unconditional basic sequence, so sometimes we will no mention this fact as in the following:

Proposition 3.1. Let $f \in E^{p}(X, F, \mu), f=\sum_{k=1}^{\infty} a_{k} f_{k}$, then

$$
\left\|\mathcal{M}^{d} f\right\|_{p} \leq C_{p}\left\|\sum_{k=1}^{\infty} a_{k} \log (k+1) f_{k}\right\|_{p}
$$

Proof. first, let us bound the difference $\sum_{N=1}^{\infty}\left\|f-S_{2^{N}} f\right\|_{p}^{p}$ :

$$
\begin{gathered}
\sum_{N=1}^{\infty}\left\|f-S_{2^{N}} f\right\|_{p}^{p}=\sum_{N=1}^{\infty}\left\|\sum_{k=2^{N}+1}^{\infty} a_{k} f_{k}\right\|_{p}^{p} \leq c_{p} \sum_{N=1}^{\infty}\left\|\left(\sum_{k=2^{N}+1}^{\infty}\left|a_{k} f_{k}\right|^{2}\right)^{\frac{1}{2}}\right\|_{p}^{p} \\
=c_{p} \sum_{N=1}^{\infty}\left\|\left(\sum_{k=2^{N}+1}^{\infty}\left|a_{k} \frac{\log (k+1)}{\log (k+1)} f_{k}\right|^{2}\right)^{\frac{1}{2}}\right\|_{p}^{p} \|_{p} \\
\leq c_{p} \sum_{N=1}^{\infty} \frac{1}{\left(\log \left(2^{N}+1\right)\right)^{p}}\left\|\left(\sum_{k=2^{N}+1}^{\infty}\left|a_{k} \log (k+1) f_{k}\right|^{2}\right)^{\frac{1}{2}}\right\|_{p}^{p} \\
\leq K \sum_{N=1}^{\infty} \frac{1}{(N+1)^{p}}\left\|\left(\sum_{k=2^{N}+1}^{\infty}\left|a_{k} \log (k+1) f_{k}\right|^{2}\right)^{\frac{1}{2}}\right\|_{p}^{p} .
\end{gathered}
$$


On the other hand, given $x \in X$ :

$$
\begin{gathered}
\left|S_{2^{N}} f(x)\right|^{p} \leq 2^{p-1}\left(|f(x)|^{p}+\left|S_{2^{N}} f(x)-f(x)\right|^{p}\right) \\
\leq 2^{p-1}\left(|f(x)|^{p}+\sum_{N=1}^{\infty}\left|S_{2^{N}} f(x)-f(x)\right|^{p}\right),
\end{gathered}
$$

then

$$
\left|\mathcal{M}^{d} f(x)\right|^{p} \leq 2^{p-1}\left(|f(x)|^{p}+\sum_{N=1}^{\infty}\left|S_{2^{N}} f(x)-f(x)\right|^{p}\right) .
$$

Integrating at both sides of the inequality,

$$
\int_{X}\left|\mathcal{M}^{d} f(x)\right|^{p} d \mu \leq 2^{p-1}\left(\int_{X}|f(x)|^{p} d \mu+\sum_{N=1}^{\infty} \int_{X}\left|S_{2^{N}} f(x)-f(x)\right|^{p} d \mu\right),
$$

then by 3.2

$$
\begin{aligned}
& \leq K\left\|\left(\sum_{k=1}^{\infty}\left|a_{k} f_{k}\right|^{2}\right)^{\frac{1}{2}}\right\|_{p}^{p}+K^{\prime}\left\|\left(\sum_{k=2^{N}+1}^{\infty}\left|a_{k} \log (k+1) f_{k}\right|^{2}\right)^{\frac{1}{2}}\right\|_{p}^{p} \\
& \leq K^{\prime \prime}\left\|\left(\sum_{k=1}^{\infty}\left|a_{k} \log (k+1) f_{k}\right|^{2}\right)^{\frac{1}{2}}\right\|_{p}^{p} \leq K^{\prime \prime \prime}\left\|\sum_{k=1}^{\infty} a_{k} \log (k+1) f_{k}\right\|_{p}^{p} .
\end{aligned}
$$

Proposition 3.2. Let $f \in L^{p}(X, F, \mu), f=\sum_{k=1}^{\infty} a_{k} f_{k}$; then

$$
\|\mathcal{M} f\|_{p}^{p} \leq C_{p}\left\|\sum_{k=1}^{\infty} a_{k} \log (k+1) f_{k}\right\|_{p}^{p}+2^{p} \sum_{N=1}^{\infty}\left\|\mathcal{M}_{N}^{\sharp} f\right\|_{p}^{p} .
$$

Proof. $\quad$ Let $x \in X$;

$$
S_{n} f(x)=\sum_{k=1}^{2^{N-1}} a_{k} f_{k}(x)+\sum_{k=2^{N-1}+1}^{n} a_{k} f_{k}(x)
$$

and then

$$
\left|S_{n} f(x)\right| \leq\left|\sum_{k=1}^{2^{N-1}} a_{k} f_{k}(x)\right|+\left|\sum_{k=2^{N-1}+1}^{n} a_{k} f_{k}(x)\right|
$$




$$
\begin{array}{r}
\left|S_{n} f(x)\right| \leq \sup _{N \geq 1}\left|S_{2^{N}} f(x)\right|+\left|\sum_{k=2^{N-1}+1}^{n} a_{k} f_{k}(x)\right|, \quad\left(n<2^{N}\right) \\
\leq \sup _{N \geq 1}\left|S_{2^{N}} f(x)\right|+\sup _{2^{N-1}<n<2^{N}}\left|\sum_{k=2^{N-1}+1}^{n} a_{k} f_{k}(x)\right| \leq \mathcal{M}^{d} f(x)+\mathcal{M}_{N}^{\sharp} f(x),
\end{array}
$$

then

$$
\begin{gathered}
\left|S_{n} f(x)\right|^{p} \leq 2^{p-1}\left(\left|\mathcal{M}^{d} f(x)\right|^{p}+\left|\mathcal{M}_{N}^{\sharp} f(x)\right|^{p}\right) \\
\leq 2^{p-1}\left(\left|\mathcal{M}^{d} f(x)\right|^{p}+\sum_{N=1}^{\infty}\left|\mathcal{M}_{N}^{\sharp} f(x)\right|^{p}\right),
\end{gathered}
$$

This last bound is independent of $n \in \mathbf{N}$, then

$$
\int_{X}|\mathcal{M} f(x)|^{p} d \mu \leq 2^{p-1}\left(\int_{X}\left|\mathcal{M}^{d} f(x)\right|^{p} d \mu+\sum_{N=1}^{\infty} \int_{X}\left|\mathcal{M}_{N}^{\sharp} f(x)\right|^{p} d \mu\right),
$$

but from equation 3.1

$$
\int_{X}|\mathcal{M} f(x)|^{p} d \mu \leq 2^{p-1}\left(c_{p}\left\|\sum_{k=1}^{\infty} a_{k} \log (k+1) f_{k}\right\|_{p}^{p}+\sum_{N=1}^{\infty} \int_{X}\left|\mathcal{M}_{N}^{\sharp} f(x)\right|^{p} d \mu\right) .
$$

In the following we will use a rather classical technique which consists in decomposing the partial sums in dyadic blocks [1], [14]. From this fact we can easily see that if we take $f \in L^{p}(X, F, \mu), f=\sum_{k=1}^{\infty} a_{k} f_{k}$ then

$$
\left\|\mathcal{M}_{N} f\right\|_{p} \leq \sum_{k=0}^{N}\left(\int_{X} \max _{1 \leq i \leq 2^{k}}\left|S_{i k} f(x)\right|^{p} d \mu\right)^{\frac{1}{p}}
$$

where $\mathcal{M}_{N} f(x)=\max _{n \leq 2^{N}}\left|S_{n} f(x)\right|$ and

$$
S_{i k} f(x)=\sum_{m=(i-1) 2^{N-k}+1}^{i 2^{N-k}} a_{m} f_{m}(x)
$$

This follows immediately from the following fact: Take $x \in X$ then there exists $n^{*}(x)$ such that $\max _{n \leq 2^{N}}\left|S_{n} f(x)\right|=\left|S_{n^{*}(x)} f(x)\right|$ but if we decompose this 
sum in dyadic blocks (of length $2^{i}, i=0,1,2 \ldots$ ) we have;

$$
\begin{gathered}
\left|\sum_{k=1}^{n^{*}(x)} a_{k} f_{k}(x)\right| \leq\left|a_{1} f_{1}(x)\right|+\left|a_{2} f_{2}(x)+a_{3} f_{3}(x)\right|+\left|a_{4} f_{4}(x)+\ldots .+a_{7} f_{7}(x)\right|+(\ldots) \\
\leq \sum_{k=0}^{N} \max _{1 \leq i \leq 2^{k}}\left|S_{i k} f(x)\right|
\end{gathered}
$$

From this last result we obtain:

Proposition 3.3. Let $p \geq 2, f \in L^{p}(X, F, \mu)$ and $f=\sum_{k=1}^{\infty} a_{k} f_{k}$ then

$$
\sum_{N=1}^{\infty}\left\|\mathcal{M}_{N}^{\sharp} f\right\|_{p}^{p} \leq K\left\|\sum_{k=1}^{\infty} a_{k} \log (k+1) f_{k}\right\|_{p}^{p} .
$$

Proof. There exists a disjoint family of sets $\left\{A_{i}\right\}_{i} \subset F$ such that

$$
\underset{1 \leq i \leq 2^{k}}{\max }\left|S_{i k} f(x)\right|=\left|\sum_{i=1}^{2^{k}} S_{i k} f(x) 1_{A_{i}}\right|,
$$

then

$$
\int_{X} \max _{1 \leq i \leq 2^{k}}\left|S_{i k} f(x)\right|^{p} d \mu \leq \sum_{i=1}^{2^{k}} \int_{X}\left|S_{i k} f(x)\right|^{p} 1_{A_{i}} d \mu .
$$

But, if the $f_{k}$ 's form an unconditional basic sequence then

$$
\begin{aligned}
& \sum_{i=1}^{2^{k}} \int_{X}\left|S_{i k} f(x)\right|^{p} 1_{A_{i}} d \mu \leq K \sum_{i=1}^{2^{k}} \int_{X}\left(\sum_{m=(i-1) 2^{N-k}+1}^{i 2^{N-k}}\left|a_{m} f_{m}(x)\right|^{2}\right)^{\frac{p}{2}} d \mu \\
& \quad \leq K \int_{X}\left(\sum_{i=1}^{2^{k}} \sum_{m=(i-1) 2^{N-k}+1}^{i 2^{N-k}}\left|a_{m} f_{m}(x)\right|^{2}\right)^{\frac{p}{2}} d \mu \quad(p \geq 2),
\end{aligned}
$$

then from equation 3.4 we have

$$
\left\|\mathcal{M}_{N} f\right\|_{p} \leq K(N+1)\|f\|_{p}
$$


In particular, replacing $f$ with $\sum_{m=2^{N-1}+1}^{2^{N}-1} a_{m} f_{m}(x)$ we get $\mathcal{M}_{N} f(x)=\mathcal{M}_{N}^{\sharp} f(x)$ and then

$$
\left\|\mathcal{M}_{N}^{\sharp} f\right\|_{p} \leq K(N+1)\left\|\sum_{m=2^{N-1}+1}^{2^{N}-1} a_{m} f_{m}\right\|_{p},
$$

from this we get

$$
\begin{gathered}
\sum_{N=1}^{\infty}\left\|\mathcal{M}_{N}^{\sharp} f\right\|_{p}^{p} \leq K^{\prime} \sum_{N=1}^{\infty}(N+1)^{p}\left\|\left(\sum_{m=2^{N-1}+1}^{2^{N}-1}\left|a_{m} f_{m}\right|^{2}\right)^{\frac{1}{2}}\right\|_{p}^{p} \|_{X} \\
=K^{\prime} \sum_{N=1}^{\infty}(N+1)^{p}\left(\sum_{m=2^{N-1}+1}^{2^{N}-1}\left|a_{m} f_{m}(x)\right|^{2}\right)^{\frac{p}{2}} d \mu \\
\leq K^{\prime} \int_{X} \sum_{N=1}^{\infty}\left(\sum_{m=2^{N-1}+1}^{2^{N}-1}\left|a_{m}(\log (m)+1) f_{m}(x)\right|^{2}\right)^{\frac{p}{2}} d \mu .
\end{gathered}
$$

Since $p \geq 2$, using a similar argument as in inequality 3.6, we have

$$
\begin{aligned}
& \leq K^{\prime} \int_{X}\left(\sum_{N=1}^{\infty} \sum_{m=2^{N-1}+1}^{2^{N}-1}\left|a_{m}(\log (m)+1) f_{m}(x)\right|^{2}\right)^{\frac{p}{2}} d \mu \\
& \leq K^{\prime \prime} \int_{X}\left(\sum_{N=1}^{\infty} \sum_{m=2^{N-1}+1}^{2^{N}-1}\left|a_{m} \log (m+1) f_{m}(x)\right|^{2}\right)^{\frac{p}{2}} d \mu,
\end{aligned}
$$

from which the result follows.

With all these results we can completely bound the maximal function $\mathcal{M} f$ and hence we can give a proof of the following:

Theorem 3.1. Let $p \geq 2$ and $\left\{f_{k}\right\}_{k}$ be an unconditional basic sequence such that

$$
\sum_{k=1}^{\infty} a_{k} \log (k+1) f_{k}
$$

converges in the norm of $L^{p}(X, F, \mu)$ then:

I) $\sum_{k=1}^{\infty} a_{k} f_{k}$ converges in $L^{p}$-norm.

II) $\sum_{k=1}^{\infty} a_{k} f_{k}(x)$ converges for almost all $x \in X[\mu]$. 
Remark Here we are considering the limit of the partial sum operators $\left.S_{k} f . \mathrm{I}\right)$ and II) in some way recover the result of [2].

Proof. Part I) is trivial.

Part II) follows from the fact that the maximal function $\mathcal{M} f$ can be bounded by combining propositions 3.2 and 3.3 :

$$
\|\mathcal{M} f\|_{p} \leq K\left\|\sum_{k=1}^{\infty} a_{k} \log (k+1) f_{k}\right\|_{p} .
$$

\section{Main Result}

In the following we will consider the case $L^{p}$, with $p \geq 2$. Let $f \in \operatorname{span}\left\{f_{j}\right\}_{j}$ , $f=\sum_{k=1}^{\infty} a_{k} f_{k}$ and consider $m_{n}: \mathbf{N} \rightarrow \mathbf{N}$ an increasing sequence: $m_{n}<$ $m_{n+1}$, then if we define $g_{n}:=\sum_{k=m_{n}+1}^{m_{n+1}} a_{k} f_{k}=S_{m_{n+1}} f-S_{m_{n}} f,\left\{g_{j}\right\}_{j}$ is again an unconditional basic sequence, with this in mind, as a direct application of theorem 3.1 we get that if $\sum_{k=1}^{\infty} a_{k} \log (k+1) g_{k}$ converges in the norm of $L^{p}(X, F, \mu)$ then $S_{m_{n}} f(x)$ converges for almost all $x \in X[\mu]$, as $n \rightarrow \infty$. In particular, if $m_{n}=2^{n}$, since the $\left\{f_{j}\right\}_{j}$ are an unconditional basic sequence:

$$
\begin{gathered}
\left\|\sum_{n=1}^{\infty} \log (n+1) \sum_{k=2^{n}+1}^{2^{n+1}} a_{k} f_{k}\right\|_{p} \leq C_{p}\left\|\left(\sum_{n=1}^{\infty}(\log (n+1))^{2} \sum_{k=2^{n}+1}^{2^{n+1}}\left|a_{k} f_{k}\right|^{2}\right)^{\frac{1}{2}}\right\|_{p} \\
\leq C_{p}\left\|\left(\sum_{n=1}^{\infty}\left(\log \log \left(2^{2 n}\right)\right)^{2} \sum_{k=2^{n}+1}^{2^{n+1}}\left|a_{k} f_{k}\right|^{2}\right)^{\frac{1}{2}}\right\|_{p} \\
\leq 2 C_{p}\left\|\left(\sum_{n=1}^{\infty} \sum_{k=2^{n}+1}^{2^{n+1}}\left|a_{k} f_{k} \log \log (k+1)\right|^{2}\right)^{\frac{1}{2}}\right\|_{p} .
\end{gathered}
$$

So we have proved the following: 
Proposition 4.1. Let $p \geq 2$ and $\left\{f_{k}\right\}_{k}$ be an unconditional basic sequence such that

$$
\sum_{k=1}^{\infty} a_{k} \log \log (k+1) f_{k}
$$

converges in the norm of $L^{p}(X, F, \mu)$, then $S_{2^{n}} f(x)$ converges for almost all $x \in X[\mu]$, as $n \rightarrow \infty$.

From this fact, we may prove the main result of this work, but as in as in [10], the proof relies in a maximal inequality which we shall prove first:

Lemma 4.1. Fixed $\left\{f_{j}\right\}_{j}$ an unconconditional basic sequence, for every $0<\varepsilon \leq 2$, there exists a constant $C(\varepsilon)$ depending only on $\varepsilon$ such that for all $\left(a_{j}\right)_{j} \in \mathbf{C}^{\mathbf{N}}$, and all $N \in \mathbf{N}$ :

$$
\int_{X} \max _{1 \leq i \leq N}\left|S_{i} f(x)\right|^{p} d \mu \leq C(\varepsilon) \log (2 N)^{\frac{\varepsilon p}{2}}\left\|\left(\sum_{i=1}^{N}\left|a_{i} f_{i}\right|^{2}\left(\log \frac{2 A^{2}}{a_{i}^{2}}\right)^{2-\varepsilon}\right)^{\frac{1}{2}}\right\|_{L^{p}}^{p},
$$

where $A^{2}=\sum_{k=1}^{N} a_{k}^{2}$

Proof. Let us proceed as in [10], consider the case $N=2^{2^{r}}=n_{r}$; $r \in \mathbf{N}$; and define $\mathbf{I}_{n_{r}}=\left\{1, \ldots, 2^{2^{r}}\right\}$ and for $p=0,1, \ldots, r-1$ set $J_{p}=$ $\left\{2^{2^{p}}+1, \ldots, 2^{2^{p+1}}\right\}$.

Now, we can find a permutation $\pi \in S_{\mathbf{I}_{n_{r}}}$ such that:

$$
\left|a_{\pi(1)}\right| \geq\left|a_{\pi(2)}\right| \geq \ldots \geq\left|a_{\pi\left(n_{r}\right)}\right|,
$$

and we can also find a permutation $\pi^{\prime} \in S_{\mathbf{I}_{n_{r}}}$ such that

$$
\pi \circ \pi^{\prime}\left(n_{p}+1\right)<\ldots<\pi \circ \pi^{\prime}\left(n_{p+1}\right)
$$

that is, a permutation such that $\pi\left(J_{p}\right)$ is reordered with the natural order of the indexes, for each $p=0, \ldots, r-1$. On the other hand, for some $N(x)$ :

$$
\begin{gathered}
\max _{1 \leq i \leq N}\left|S_{i} f(x)\right|=\left|S_{N(x)} f(x)\right| \\
\leq\left|a_{\pi(1)} f_{\pi(1)}(x)\right|+\left|a_{\pi(2)} f_{\pi(2)}(x)\right|+\sum_{s=0}^{r-1}\left|\sum_{i \in J_{s}} a_{\pi \circ \pi^{\prime}(i)} f_{\pi \circ \pi^{\prime}(i)}(x) 1_{\left\{k: \pi \circ \pi^{\prime}(k) \leq N(x)\right\}}\right|
\end{gathered}
$$




$$
\leq\left|a_{\pi(1)} f_{\pi(1)}(x)\right|+\left|a_{\pi(2)} f_{\pi(2)}(x)\right|+\sum_{s=0}^{r-1} \max _{k \in J_{s}}\left|\sum_{i=n_{s}+1}^{k} a_{\pi \circ \pi^{\prime}(i)} f_{\pi \circ \pi^{\prime}(i)}(x)\right| .
$$

Taking the $p$ norm at each side of the inequality and by the triangle inequality:

$$
\left(\int_{X} \max _{1 \leq i \leq N}\left|S_{i} f(x)\right|^{p} d \mu\right)^{\frac{1}{p}} \leq\left|a_{\pi(1)}\right|\left\|f_{\pi(1)}\right\|_{p}+\left|a_{\pi(2)}\right|\left\|f_{\pi(2)}\right\|_{p}+
$$

$$
+\sum_{s=0}^{r-1}\left(\int_{X}\left(\max _{k \in J_{s}}\left|\sum_{i=n_{s}+1}^{k} a_{\pi \circ \pi^{\prime}(i)} f_{\pi \circ \pi^{\prime}(i)}(x)\right|\right)^{p} d \mu\right)^{\frac{1}{p}} .
$$

Apply the maximal inequality 3.8 obtained in the proof of theorem 3.1 to the finite sum to get:

$$
\begin{gathered}
\left(\int_{X} \max _{1 \leq i \leq N}\left|S_{i} f(x)\right|^{p} d \mu\right)^{\frac{1}{p}} \leq\left|a_{\pi(1)}\right|\left\|f_{\pi(1)}\right\|_{p}+\left|a_{\pi(2)}\right|\left\|f_{\pi(2)}\right\|_{p}+ \\
+K \sum_{s=0}^{r-1}\left(\int_{X}\left(\sum_{i=n_{s}+1}^{n_{s+1}}\left|a_{\pi(i)} f_{\pi(i)}(x) \log (i+1)\right|^{2}\right)^{\frac{p}{2}} d \mu\right)^{\frac{1}{p}} .
\end{gathered}
$$

Now, if $i \leq 2^{2^{s}}$ then $\log (i+1)^{\epsilon} \leq 4^{\frac{\epsilon}{2}} 2^{s \epsilon}$, so that:

$$
\begin{aligned}
& \sum_{s=0}^{r-1} 4^{\frac{\epsilon}{2}} 2^{\frac{s \epsilon}{2}}\left(\int_{X}\left(\sum_{i=n_{s}+1}^{n_{s+1}}\left|a_{\pi(i)} f_{\pi(i)}(x) \log (i+1)\right|^{2}\right)^{\frac{p}{2}} d \mu\right)^{\frac{1}{p}} \\
& \leq \sum_{s=0}^{r-1}\left(\int_{X}\left(\sum_{i=n_{s}+1}^{n_{s+1}}\left|a_{\pi(i)} f_{\pi(i)}(x)\right|^{2}(\log (i+1))^{2-\epsilon}\right)^{\frac{p}{2}} d \mu\right)^{\frac{1}{p}}
\end{aligned}
$$

$$
\leq\left(\sum_{s=0}^{r-1} 4^{\frac{\epsilon q}{2}} 2^{\frac{s \epsilon q}{2}}\right)^{\frac{1}{q}}\left(\sum_{s=0}^{r-1} \int_{X}\left(\sum_{i=n_{s}+1}^{n_{s+1}}\left|a_{\pi(i)} f_{\pi(i)}(x)\right|^{2}|\log (i+1)|^{2-\epsilon}\right)^{\frac{p}{2}} d \mu\right)^{\frac{1}{p}},
$$


by Hölder's inequality, with $\frac{1}{p}+\frac{1}{q}=1$. Since $p \geq 2$ then 4.3 is less than:

$$
4^{\frac{\epsilon}{2}}\left(\frac{2^{\frac{r \epsilon q}{2}}}{2^{\frac{\epsilon q}{2}}-1}\right)^{\frac{1}{q}}\left(\int_{X}\left(\sum_{i=3}^{n_{r}}\left|a_{\pi(i)} f_{\pi(i)}(x)\right|^{2}|\log (i+1)|^{2-\epsilon}\right)^{\frac{p}{2}} d \mu\right)^{\frac{1}{p}}
$$

Since $\forall k \in J_{r} \cup\{1,2\}: k\left|a_{\pi(k)}\right|^{2} \leq \sum_{i=1}^{n_{r}}\left|a_{\pi(i)}\right|^{2}$, if we write $A^{2}{ }_{n_{r}}=$ $\sum_{i=1}^{n_{r}}\left|a_{\pi(i)}\right|^{2}$ then 4.4 is less than:

$$
\leq 4^{\frac{\epsilon}{2}}\left(\frac{2^{\frac{r \epsilon q}{2}}}{2^{\frac{\epsilon q}{2}}-1}\right)^{\frac{1}{q}}\left(\int_{X}\left(\sum_{i=3}^{n_{r}}\left|a_{\pi(i)} f_{\pi(i)}(x)\right|^{2}\left(\log \frac{2 A^{2} n_{r}}{\left|a_{\pi(i)}\right|^{2}}\right)^{2-\epsilon}\right)^{\frac{p}{2}} d \mu\right)^{\frac{1}{p}} .
$$

Combining inequalities 4.2 and 4.5 we obtain:

$$
\begin{gathered}
\left(\int_{X} \max _{1 \leq i \leq N}\left|S_{i} f(x)\right|^{p} d \mu\right)^{\frac{1}{p}} \leq C(\epsilon) \log \left(n_{r}+1\right)^{\frac{\epsilon}{2}} \times(\ldots) \\
(\ldots) \times\left(\int_{X}\left(\sum_{i=3}^{n_{r}}\left|a_{\pi(i)} f_{\pi(i)}(x)\right|^{2}\left(\log \frac{2 A^{2}{ }_{n_{r}}}{\left|a_{\pi(i)}\right|^{2}}\right)^{2-\epsilon}\right)^{\frac{p}{2}} d \mu\right)^{\frac{1}{p}} .
\end{gathered}
$$

Now we can give a proof for theorem 1.1:

Proof. (Theorem 1.1) Write $A^{2}{ }_{N}=\sum_{i \in I_{N}}\left|a_{i}\right|^{2}$, where $I_{N}=\left\{2^{N}+\right.$ $\left.1, \ldots, 2^{N+1}\right\}$. Using lemma 4.1 we can estimate as in 3.3 .

$$
\left\|\mathcal{M}_{N}^{\sharp} f\right\|_{p}^{p} \leq C(\epsilon)(N+1)^{\frac{\epsilon p}{2}}\left\|\left(\sum_{k \in I_{N-1}}\left|a_{k} f_{k}\right|^{2}\left(\log \frac{2 A^{2}{ }_{N-1}}{\left|a_{k}\right|^{2}}\right)^{2-\epsilon}\right)^{\frac{1}{2}}\right\|_{p}^{p} .
$$




$$
\begin{gathered}
\sum_{N=1}^{\infty}\left\|\mathcal{M}_{N}^{\sharp} f\right\|_{p}^{p} \leq C(\epsilon) \sum_{N=1}^{\infty}\left\|\left((N+1)^{\epsilon} \sum_{k \in I_{N-1}}\left|a_{k} f_{k}\right|^{2}\left(\log \frac{2 A^{2}{ }_{N-1}}{\left|a_{k}\right|^{2}}\right)^{2-\epsilon}\right)^{\frac{1}{2}}\right\|_{p}^{p} \\
\leq 2 C(\epsilon) \sum_{N=1}^{\infty}\left\|\left(\sum_{k \in I_{N-1}}\left|a_{k} f_{k}\right|^{2}\left(\log \frac{2 A^{2}{ }_{N-1}}{\left|a_{k}\right|^{2}}\right)^{2-\epsilon} \log (k)^{\epsilon}\right)^{\frac{1}{2}}\right\|_{p}^{p} \|_{p} \\
\leq 2 C(\epsilon)\left\|\left(\sum_{N=1}^{\infty} \sum_{k \in I_{N-1}}\left|a_{k} f_{k}\right|^{2}\left(\log \frac{2 A^{2}{ }_{N-1}}{\left|a_{k}\right|^{2}}\right)^{2-\epsilon} \log (k)^{\epsilon}\right)^{\frac{1}{2}}\right\|_{p}^{p}
\end{gathered}
$$

( $p \geq 2$ and Monotone Convergence)

Then under the hypothesis of theorem 1.1 we have:

$$
\sum_{N=1}^{\infty}\left\|\mathcal{M}_{N}^{\sharp} f\right\|_{p}^{p}<\infty
$$

Now, we can proceed as in theorem 3.1 or we can use equation 4.7 to obtain:

$$
\lim _{N \rightarrow \infty} \mathcal{M}_{N}^{\sharp} f(x)=0 .
$$

On the other hand the (unconditional) convergence in $L^{p}$ norm of

$$
\sum_{n=0}^{\infty} \sum_{j \in I_{n}} a_{j} \log (j)^{\frac{\epsilon}{2}}\left(\log \frac{2 A_{n}^{2}}{a_{j}^{2}}\right)^{1-\frac{\epsilon}{2}} f_{j},
$$

implies the convergence in $L^{p}$ norm of

$$
\sum_{k=1}^{\infty} a_{k} \log \log (k+1) f_{k} .
$$

As a consequence of proposition 4.1, and since given $m \in \mathbf{N}$ and $2^{m-1}<$ $n<2^{m}$ for almost all $x \in X$ the following holds:

$$
\left|S_{n} f(x)-f(x)\right| \leq \mathcal{M}_{m+1}^{\sharp} f(x)+\left|S_{2^{m}} f(x)-f(x)\right|
$$

where $f=\sum_{k=1}^{\infty} a_{k} f_{k}\left(L^{p}\right)$, then by proposition $4.1, S_{2^{m}} f(x)$ converges to $f(x)[\mu]$-a.e. as $m \rightarrow \infty$, and on the other hand $\mathcal{M}_{m+1}^{\sharp} f(x) \rightarrow 0$, so that the proof is complete. 


\section{References}

[1] Alexits G. , Convergence Problems of Orthogonal Series, Pergamon Press, (1961).

[2] Bennett, G. Unconditional Convergence and Almost Everywhere Convergence Z. Wahrs. verw. Gebeite Vol. 34, pp. 135-155, (1976).

[3] Gerre, S., Classical Sequences in Banach Spaces, Marcel Dekker, (1992).

[4] Houdré C. On the almost sure convergnece of series of satationary and related nonstationary variables, Ann. of Prob. Vol. 23 (3), pp. 12041218, (1985).

[5] Kahane J. P. Some Random Series of Functions, Cambridge, (1993).

[6] Lindenstrauss J. Tzafriri L. Classical Banach Spaces, Vol. I y II, Springer Verlag 2ed., (1996).

[7] Loéve M., Probability Theory, Vol. I, Springer Verlag, (1977).

[8] Medina J. M. , Cernuschi -Frías B. Random series in $L^{p}(X, \Sigma, \mu)$ using Unconditional Basic Sequences and $l^{p}$ stable sequences: A result on almost sure almost everywhere convergence, Proc. A. M. S. Vol.135 (11), pp. 3561-3569, (2007).

[9] Menchoff D. Sur les séries de fonctions orthogonales I., Fund. Math. 4, 1923, pages 82-105. Vol. 40 (2), September, pp. 1490-1503, (1994).

[10] Móricz F., Tándori K. An Improved Menshov-Rademacher Theorem, Proc. A. M. S. Vol. 124 (3), pp. 877-885, (1996).

[11] Ø̈rno P. A note on Unconditionally converging series in $L^{p}$, Proc. A. M. S. Vol. 59 (2), 252-254, (1976). Lecture Notes in Mathematics No. 672 , Springer-Verlag, (1978).

[12] Wojtaszczyk P. Banach Spaces for Analysts, Cambridge, (1996).

[13] Yang L., Unconditional Basic Sequence in $L^{p}(\mu)$ and its $l^{p}$ stability, Proc. A. M. S. Vol. 127(2), pp. 455-464, (1999).

[14] Zygmund A., Trigonometric Series, Vol II. Cambridge, (1958). 
Juan M. Medina

Departamento de Matemática, Instituto Argentino de Matemática, Universidad de Buenos Aires, Paseo Colón 850 (1063)

Capital Federal CONICET,

Argentina

e-mail : jmedina@fi.uba.ar

and

Bruno Cernuschi-Frías

Facultad de Ingeniería,

Instituto Argentino de Matemática, Universidad de Buenos Aires,

Paseo Colón 850 (1063)

Capital Federal

CONICET,

Argentina

e-mail : bcf@ieee.org 